\title{
Transcranial Magnetic Stimulation for the Treatment of Cocaine Addiction: A Systematic Review
}

\author{
Alezandra Torres-Castaño ${ }^{1,2,3, *}$, Amado Rivero-Santana ${ }^{1,2}$, Lilisbeth Perestelo-Pérez ${ }^{2}$, Andrea Duarte-Díaz ${ }^{1,2}$, \\ Ana Toledo-Chávarri ${ }^{1,2}$, Vanesa Ramos-García 1,2 (D), Yolanda Álvarez-Pérez ${ }^{1,2}$, Javier Cudeiro-Mazaira ${ }^{4,5}$, \\ Iván Padrón-González ${ }^{6} \mathbb{1}$ and Pedro Serrano-Pérez ${ }^{7}$
}

check for updates

Citation: Torres-Castaño, A.;

Rivero-Santana, A.; Perestelo-Pérez,

L.; Duarte-Díaz, A.; Toledo-Chávarri,

A.; Ramos-García, V.; Álvarez-Pérez,

Y.; Cudeiro-Mazaira, J.;

Padrón-González, I.; Serrano-Pérez, P.

Transcranial Magnetic Stimulation for the Treatment of Cocaine Addiction: A Systematic Review. J. Clin. Med. 2021, 10, 5595. https://doi.org/ $10.3390 /$ jcm10235595

Academic Editors: Haidar S. Dafsari and Rubens Gisbert Cury

Received: 27 October 2021

Accepted: 26 November 2021

Published: 28 November 2021

Publisher's Note: MDPI stays neutral with regard to jurisdictional claims in published maps and institutional affiliations.

Copyright: (C) 2021 by the authors. Licensee MDPI, Basel, Switzerland. This article is an open access article distributed under the terms and conditions of the Creative Commons Attribution (CC BY) license (https:// creativecommons.org/licenses/by/ $4.0 /)$.
1 Canary Islands Health Research Institute Foundation (FIISC), 38109 El Rosario, Spain; amado.riverosantana@sescs.es (A.R.-S.); andrea.duartediaz@sescs.es (A.D.-D.); anatoledochavarri@sescs.es (A.T.-C.); vanesa.ramosgarcia@sescs.es (V.R.-G.); yolanda.alvarezperez@sescs.es (Y.Á.-P.)

2 Evaluation Unit of the Canary Islands Health Service (SESCS), 38019 El Rosario, Spain; lilisbeth.peresteloperez@sescs.es

3 The Spanish Network of Agencies for Health Technology Assessment and Services of the National Health System (RedETS), 28071 Madrid, Spain

4 Galician Brain Stimulation Center, 15009 A Coruña, Spain; javier.cudeiro@udc.es

5 Neuroscience and Motor Control Group (NEUROcom), Instituto Biomédico de A Coruña (INIBIC), Universidad de A Coruña, 15006 Oza, Spain

6 Institute of Neuroscience, University of La Laguna, Guajara Campus, 38200 San Cristobal de La Laguna, Spain; ivpadron@ull.edu.es

7 Group of Psychiatry, Mental Health and Addictions at the Vall d'Hebron Institut de Recerca (VHIR), 08035 Barcelona, Spain; pedrogserrano@gmail.com

* Correspondence: atorrcas@sescs.es

\begin{abstract}
Long-term cocaine use is associated with cognitive deficits and neuro-psychiatric pathologies. Repetitive transcranial magnetic stimulation (rTMS) is an emerging therapeutic strategy relating to changes in brain activity. It stimulates the prefrontal cortex and is involved in inhibitory cognitive control, decision making and care. This systematic review aims to evaluate and synthesize the evidence on the safety, effectiveness, and cost-effectiveness of rTMS for the treatment of cocaine addiction. A systematic review of the literature was carried out. The following electronic databases were consulted from inception to October 2020: MEDLINE, Embase, CINAHL, PsycINFO, Cochrane Central Register of Controlled Trials and Web of Science. Randomised controlled trials, non-randomised controlled trials and case-series and full economic evaluations were included. Twelve studies were included. No identified study reported data on cost-effectiveness. Significant results of the efficacy of TMS have been observed in terms of the reduction of craving to consume and the number of doses consumed. No serious adverse effects have been observed. Despite the low quality of the studies, the first results were observed in terms of reduction of cocaine use and craving. In any case, this effect is considered moderate. Studies with larger sample sizes and longer follow-ups are required.
\end{abstract}

Keywords: cocaine use disorder; craving; non-invasive brain stimulation; transcranial magnetic stimulation; systematic review

\section{Introduction}

Cocaine use disorder (CUD) is a significant health problem, with about 12-21 million users worldwide in 2014 [1,2]. Chronic cocaine use can cause damage and changes to the prefrontal cortex (PFC) [3], including a significant reduction in brain volume [4,5], cortical hypoactivity [6,7], impaired executive functions, and dysregulation of neurotransmitter systems [8-10]. Preclinical studies have shown that loss of inhibitory control, resulting from damage to the PFC, appears to be crucial in compulsive drug-seeking behaviours [11,12] and intense and uncontrollable craving from consuming a substance [13]. This desire is one 
of the key characteristics of substance dependence, which has been shown to be one of the most important contributors to relapse. Several types of evidence indicate that substance dependence involves the dopaminergic system, causing a hypodopaminergic state in the mesolimbic system [14].

Previous research has described the neural network distributed in the two hemispheres present in the pathophysiology of craving, involving the nucleus accumbens, the amygdala, the anterior cingulum, the orbitofrontal cortex and the dorsolateral prefrontal (DLPFC) $[15,16]$. The DLPFC, specifically, participates in the reward, motivation and decision-making circuits that provide the substrate for the integration of cognitively and motivationally relevant information and the inhibitory control over the options of an immediate reward [17]. However, the poor functioning of the DLPFC and the anterior cingulate cortex may explain a reduction in inhibitory control of behaviour and a tendency to relapse into the use of alcohol and other drugs [18]. In fact, the most recent technological advances, using optogenetic techniques that allow the manipulation of neuronal groups in a very effective and localized way, have made it possible to delineate the cortico-subcortical circuits that are related to addictions in animals $[12,19]$. In these experiments, a hypofunction of the prefrontal cortex was related to a lack of subcortical inhibitory control (over the reward circuits, see Section 4).

Following this hypothesis, Terraneo et al. [13] designed an experiment in which the activation of the left dorsolateral prefrontal cortex, by means of rTMS, could, in patients addicted to cocaine, reduce consumption and craving.

To date, an effective treatment for cocaine addiction has not been found [20], and currently there are new treatments in experimental research [21]. Neuromodulation techniques, such as transcranial magnetic stimulation (TMS), have been investigated as potential treatments with fewer side-effects and contraindications than drugs for substance use disorders, and is therefore a promising therapeutic alternative to conventional pharmacotherapy and behaviour modification therapies [22].

TMS is a non-invasive human brain neuromodulation technology based on the principle of electromagnetic induction. The transient application of an electric current to a coil of conductive material produces a time-varying magnetic field, capable of inducing an electric field at a distance, affecting the electrical activity of neurons in the cerebral cortex [23,24]. This electric field must be of sufficient magnitude for neuronal depolarization to occur, followed by an increase in synaptic activity and the production of action potentials [25]. The extent of the induced field depends on the geometry and size of the coil used in the TMS equipment.

Several coils with different shapes have been designed to stimulate different regions of the brain, the most common being circular coils, which allow large areas of the cortex to be affected, and those with a figure of eight allowing more focal stimulation. Both coils are useful for superficial cortical stimulation (about 2-3 cm deep) [26]. However, in certain situations it may be useful to reach deeper targets, for which special coils have been developed, such as double cone coils that can reach a depth of 3-4 cm and the so-called $\mathrm{H}$-type coils, which can reach depths up to $6 \mathrm{~cm}$ [27]. This is what is known as deep brain stimulation TMS (dTMS). Currently, several manufacturers provide coils with specific characteristics to achieve a focused or deep stimulation [28].

Repetitive TMS (rTMS) at low frequency $(\leq 1 \mathrm{~Hz})$ has inhibitory effects [29], whereas high frequency rTMS $(>5 \mathrm{~Hz})$ is excitatory $[13,30]$. The rTMS uses a coil held against the scalp and located over the cortical zone of choice. Stimulation is performed using repetitive stimulation in the form of continuous pulses at a certain frequency, or repetitive trains of magnetic pulses which affect a specific area of the brain and those other areas that are connected [22]. Stimulation parameters of rTMS can vary significantly with respect to stimulus intensity, total number of pulses, and frequency. These variations aim to customize rTMS parameters and improve inhibitory processes, which can be abnormal in substance abuse cases (i.e., lack of impulse control or impulsivity). On the other hand, rTMS has been shown to be highly effective in studying the excitation-inhibition balance in a specific area 
of the brain. In order to do this, different cortical inhibition and excitation protocols have been developed with single-pulse TMS or paired pulses [31,32].

Different temporal patterns of stimulation have been developed. This is the case of Theta Burst Stimulation (TBS) [33], which involves bursts of three pulses at $50 \mathrm{~Hz}$ that are repeated at theta frequency $(5 / \mathrm{s})$. There are two types of TBS with opposite effects: intermittent TBS (iTBS) has an excitatory effect that lasts over time. This pattern is repeated for $190 \mathrm{~s}$, implying that the subject is supplied with 600 pulses in total [33,34]. The other protocol is known as continuous TBS (cTBS), which induces inhibitory effects that also last over time, involving a transient depression of neuronal behaviour long-term. In this case the stimulation involves a 40-s train of uninterrupted TBS (600 pulses) [15,33]. rTMS in any of its forms is a painless procedure and its common side effects, if they occur, are generally minor, although seizures have been reported very occasionally; thus, most patients tolerate it very well $[35,36]$. Consequently, rTMS has been suggested as a possible alternative treatment for substance use disorders, such as cocaine, which is currently being investigated [22].

This systematic review (SR) aims to evaluate and synthesize the evidence on the safety, effectiveness, and cost-effectiveness of TMS for the treatment of cocaine addiction.

\section{Materials and Methods}

An SR of the literature was carried out in accordance with the Preferred Reporting Items for Systematic reviews and Meta-Analysis (PRISMA) Statement [37]. The detail of the PRISMA checklist can be found in Table S1. This SR was registered in the International Prospective Register of Systematic Reviews (PROSPERO) with the number CRD42021233283.

\subsection{Search Strategy}

The following electronic databases were consulted, from inception to October 21st 2020: MEDLINE, Embase, CINAHL, PsycINFO, Cochrane Central Register of Controlled Trials and Web of Science (WOS). In addition, a manual consultation of references in non-indexed health journals and other relevant health websites was performed. Search terms were grouped around the following terms: "cocaine use disorder, substance abuse disorders, craving, transcranial magnetic stimulation". As an example, the MEDLINE search strategy is shown in Table 1. Search strategies for the other five electronic databases are available in Table S2. No language or publication year restrictions were applied to limit the search.

Table 1. Medline search strategy.

\begin{tabular}{ccc}
\hline 1 & Transcranial Magnetic Stimulation & 11,631 \\
2 & (Transcranial adj1 magnetic stimulation\$).tw. & 14,512 \\
3 & $($ (transcranial magnetic stimulation or tms) adj5 repetitive).tw. & 4917 \\
4 & $($ transcranial magnetic stimulation or tms) adj5 rhythmic).tw. & 41 \\
5 & (rtms or tms).tw. & 15,423 \\
6 & ((Repetitive or "single pulse" or "paired pulse") adj1 “transcranial magnetic & 5260 \\
7 & stimulation").tw. & 21,895 \\
8 & 1 or 2 or 3 or 4 or 5 or 6 & 8278 \\
9 & Cocaine-Related Disorders & 7622 \\
10 & (cocaine * adj2 (abuse* or addict * or dependent *or disorder *)). ti,ab. & 12,519 \\
11 & 8 or 9 & 46 \\
\hline
\end{tabular}

Additionally, manual searches were carried out on clinical trail.com to identify ongoing studies. 


\subsection{Inclusion and Exclusion Criteria}

\subsubsection{Design}

Randomised controlled trials (RCTs), non-randomised controlled trials (nRCTs), caseseries and full economic evaluations (EE) published in English or Spanish were included. Qualitative studies, conference abstracts, letters, commentaries, essays, and book chapters were excluded.

\subsubsection{Population}

Studies addressing subjects with cocaine dependence or CUD, seeking treatment or not, were included. Thus, studies with participants taking opioids, cannabis, tobacco, alcohol, and food abusers or non-abusers were excluded. Studies with mixed use were included as long as the effect on cocaine use and craving was evaluated, and the results were reported separately.

\subsubsection{Intervention}

Studies applying any rTMS protocol were included. Studies with a single session were excluded, unless they used more recent stimulation protocols such as deep rTMS or TBS.

\subsubsection{Comparator}

The main comparators considered were sham-stimulation, waiting list, pharmacological treatment, no treatment and treatment as usual.

\subsubsection{Outcomes}

The primary effectiveness outcomes were the reduction of cocaine use or relapses, evaluated by laboratory analyses (e.g., urine, hair), self- and hetero report. Secondary outcomes included reduction of craving, addiction severity, anxiety, depression, and sleep quality. Regarding, safety, the main outcomes were serious and non-serious adverse events. The incremental cost-effectiveness ratio (ICER) was the cost-effectiveness outcome.

\subsection{Study Selection}

Bibliographic references were stored using the Reference Manager Edition Version $10^{\odot}$ (Thomson Scientific, EE.UU.). Electronic search results were downloaded into a standardized Excel datasheet and duplicates were removed. Titles and abstracts were screened first. Subsequently, those articles selected as relevant were full text reviewed to determine whether a study met the inclusion criteria. This screening process was conducted independently by two reviewers. Any doubt or disagreement was resolved by discussion and, when necessary, with the participation of a third reviewer. The selection process and the reasons for full-text exclusion were recorded and documented in a PRISMA flow diagram [37].

\subsection{Data Extarction and Analysis}

The following items were extracted based on a previously designed Excel form: author, year, country, study design, participant's characteristics, stimulation protocol, frequency and intensity, stimulation area, comparator, main outcomes, and follow-up. If relevant missing data was identified, the corresponding author was contacted and asked to provide the missing details. Data extraction was performed by one reviewer and checked by another. Any discrepancies were resolved through discussion. Given the high heterogeneity of the methods, protocols and stimulation area within the studies, meta-analysis was not carried-out and, thus, the results were merged and described narratively.

\subsection{Quality Assessment}

The risk of bias of the included studies were assessed with the Cochrane risk-of-bias tool for (RoB 2) [38] for RCTs, the Joanna Briggs Institute (JBI) checklist [39] for nRCT, and the Institute of Health Economics (IHE) quality appraisal checklist for case-series [40]. 
Quality assessment was performed independently by two reviewers and disagreements were solved by discussion or after consulting a third reviewer.

\section{Results}

A total of 353 studies were identified in the electronic databases. After removing duplicates, titles, and abstracts, 200 references were screened and 29 full-text articles were assessed for eligibility. Finally, 12 studies were included in this SR [13,15,17,20,41-48]. A list of ongoing studies can be found in Table S3. Figure 1 shows the PRISMA flowchart of the study selection process.

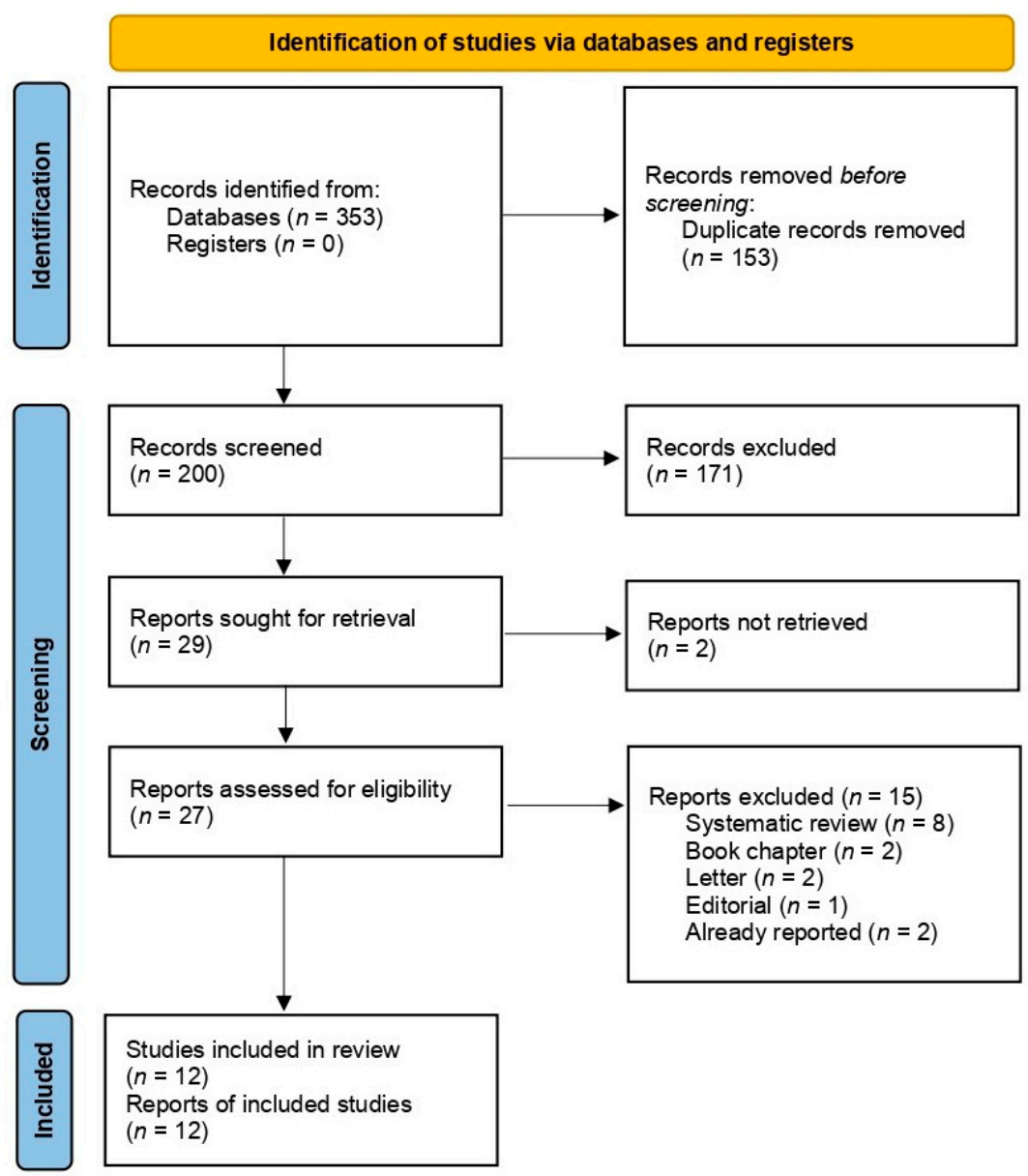

Figure 1. PRISMA flowchart of the study selection process.

\subsection{Study Characteristics}

Table 2 shows the selected studies' characteristics. Five of the included studies were RCT [13,15,41,42,44], one was an nRCT [47] and six were case-series [17,20,43,45,46,48]. None of the studies identified by this SR reported data on cost-effectiveness. Two different therapeutic protocols were identified within the studies: conventional high frequency $(10-15 \mathrm{~Hz})[13,17,20,41,43-46]$ and continuous or intermittent TBS [15,41,42,44]. Sample sizes ranged from 11-147 (median $=22.5)$. Stimulation areas were the prefrontal cortex (PFC) [47], bilateral PFC [41], medial PFC (MPFC) [15,44,46], left dorsolateral prefrontal cortex (DLPFC) $[13,17,43-46,48]$ and cingulate cortex [44]. Intervention times fluctuated in a range of one to four weeks and follow-ups varied from one hour to eight months. 
Table 2. Characteristics of the included studies.

\begin{tabular}{|c|c|c|c|c|c|c|c|c|c|c|}
\hline $\begin{array}{l}\text { Author (Year), } \\
\text { Country }\end{array}$ & Study Design & Population & $\begin{array}{c}\text { No. of } \\
\text { Participants }\end{array}$ & Intervention & $\begin{array}{l}\text { Stimulation } \\
\text { Area }\end{array}$ & $\begin{array}{l}\text { Stimulation } \\
\text { Protocol }\end{array}$ & $\begin{array}{c}\text { Frequency (Hz) } \\
\text { and Intensity } \\
(\% \text { RMT) }\end{array}$ & Comparator & Outcomes & Measures \\
\hline $\begin{array}{c}\text { Bolloni } \\
\text { (2016) [41], Italy, } \\
\text { USA and Israel }\end{array}$ & $\mathrm{RCT}$ & $\begin{array}{l}\text { CUD (DSM-5) } \\
\text { Male: } n=16 \\
\text { Female: } n=2 \\
\text { Mean age: } 27-48 \\
\text { years }\end{array}$ & $n=18$ & Deep rTMS & Bilateral PFC & $\begin{array}{l}12 \text { rTMS sessions } \\
\text { were administered } \\
\text { three times a week } \\
\text { for } 4 \text { weeks }\end{array}$ & $\begin{array}{c}10 \mathrm{~Hz} \\
100-120 \%\end{array}$ & Sham & $\begin{array}{c}\text { Cocaine intake } \\
\text { (hair analysis) }\end{array}$ & $\begin{array}{c}\text { Baseline, after } 1 \\
\text { month and } 3 \\
\text { and } 6 \text { months } \\
\text { later }\end{array}$ \\
\hline $\begin{array}{c}\text { Gómez (2020) } \\
\text { [20], Italy }\end{array}$ & CS & $\begin{array}{c}\text { CUD (DSM-5) } \\
\text { Male: } n=85 \\
\text { Female: } n=2 \\
\text { Mean age: } 37.67 \\
\text { years }\end{array}$ & $n=87$ & rTMS & Left-DLPFC & $\begin{array}{l}2 \text { sessions per day } \\
\text { for the first } 5 \\
\text { consecutive days } \\
\text { of treatment (10 } \\
\text { sessions), and } 2 \\
\text { sessions per week } \\
\text { for the following } \\
12 \text { weeks. }\end{array}$ & $\begin{array}{l}15 \mathrm{~Hz} \\
100 \%\end{array}$ & NA & $\begin{array}{c}\text { Cocaine use } \\
\text { (self-report and } \\
\text { urine screens) } \\
\text { Craving (CCQ) } \\
\text { Sleep Quality } \\
\text { (PSQI) } \\
\text { Depression } \\
\text { (BDI-II) } \\
\text { Anxiety (SAS) } \\
\text { Symptoms } \\
\text { (SCL-90) }\end{array}$ & $\begin{array}{l}\text { Baseline, and } \\
\text { after } 5,30,60, \\
\text { and } 90 \text { days of } \\
\text { rTMS treatment }\end{array}$ \\
\hline $\begin{array}{c}\text { Hanlon (2015) } \\
\text { [15], } \\
\text { USA }\end{array}$ & $\mathrm{RCT}$ & $\begin{array}{c}\text { Cocaine users } \\
\text { Male: } n=9 \\
\text { Female: } n=2 \\
\text { Mean age: } 39 \\
\text { years }\end{array}$ & $n=11$ & cTBS & MPFC & $\begin{array}{l}2 \text { stimulation } \\
\text { visits (occurring } \\
\text { within } 7-14 \text { days } \\
\text { of each other). }\end{array}$ & $\begin{array}{l}5 \mathrm{~Hz} \\
110 \%\end{array}$ & Sham & Craving (VAS) & $\begin{array}{l}\text { Before and after } \\
\text { the cTBS sessior }\end{array}$ \\
\hline $\begin{array}{c}\text { Hanlon (2017) } \\
\text { [42] } \\
\text { USA }\end{array}$ & $\mathrm{RCT}$ & $\begin{array}{c}\text { Cocaine users } \\
\text { Male: } n=12 \\
\text { Female: } n=3 \\
\text { Mean age: } 42 \\
\text { years }\end{array}$ & $n=25$ & cTBS & MPFC & $\begin{array}{l}2 \text { stimulation } \\
\text { visits (occurring } \\
\text { within } 7-14 \text { days } \\
\text { of each other) with } \\
\text { exposure to } 6 \\
\text { trains of cBTS. }\end{array}$ & $\begin{array}{l}5 \mathrm{~Hz} \\
110 \%\end{array}$ & Sham & Craving (VAS) & $\begin{array}{l}\text { Before and after } \\
\text { the cTBS sessior }\end{array}$ \\
\hline $\begin{array}{c}\text { Madeo (2020) } \\
\text { [43], } \\
\text { Italy and USA }\end{array}$ & CS & $\begin{array}{c}\text { CUD (DSM-5) } \\
\text { Male: } n=139 \\
\text { Female: } n=8 \\
\text { Mean age: } 36.6 \\
\text { years }\end{array}$ & $n=147$ & rTMS & Left-DLPFC & $\begin{array}{l}2 \text { rTMS sessions } \\
\text { per day for the } \\
\text { first } 5 \text { days, then } \\
\text { weekly, twice per } \\
\text { day on each } \\
\text { session day for } 11 \\
\text { consecutive } \\
\text { weeks. }\end{array}$ & $\begin{array}{l}15 \mathrm{~Hz} \\
100 \%\end{array}$ & NA & $\begin{array}{l}\text { Cocaine use } \\
\text { (urine screening, } \\
\text { self-report and } \\
\text { reports by } \\
\text { collateral } \\
\text { informants) }\end{array}$ & $\begin{array}{c}\text { Baseline, after } 3 \\
\text { months of rTMS } \\
\text { and up to } 2 \\
\text { years. }\end{array}$ \\
\hline
\end{tabular}


Table 2. Cont.

\begin{tabular}{|c|c|c|c|c|c|c|c|c|c|c|}
\hline $\begin{array}{l}\text { Author (Year), } \\
\text { Country }\end{array}$ & Study Design & Population & $\begin{array}{c}\text { No. of } \\
\text { Participants }\end{array}$ & Intervention & $\begin{array}{l}\text { Stimulation } \\
\text { Area }\end{array}$ & $\begin{array}{l}\text { Stimulation } \\
\text { Protocol }\end{array}$ & $\begin{array}{c}\text { Frequency (Hz) } \\
\text { and Intensity } \\
(\% \text { RMT) }\end{array}$ & Comparator & Outcomes & Measures \\
\hline $\begin{array}{l}\text { Martínez (2018) } \\
\text { [44], USA and } \\
\text { Israel }\end{array}$ & $\mathrm{RCT}$ & $\begin{array}{c}\text { CUD (DSM-5) } \\
\text { Male: } n=17 \\
\text { Female: } n=1 \\
\text { Mean age: } 43.3 \\
\text { years }\end{array}$ & $n=18$ & rTMS & MPFC & $\begin{array}{l}\text { The rTMS was } \\
\text { delivered on } \\
\text { weekdays, over } \\
\text { the course of } 3 \\
\text { weeks. }\end{array}$ & $\begin{array}{l}\text { HF: } 10 \mathrm{~Hz} \\
\text { LF: } 1 \mathrm{~Hz} \\
90-110 \%\end{array}$ & Sham & $\begin{array}{c}\text { Number of } \\
\text { doses chosen } \\
\text { during self- } \\
\text { administration } \\
\text { Craving (VAS) }\end{array}$ & $\begin{array}{c}\text { Baseline, after } 4 \\
\text { days and after } \\
13 \text { days of rTMS }\end{array}$ \\
\hline $\begin{array}{c}\text { Pettorruso } \\
\text { (2019) [45], Italy } \\
\text { and UK }\end{array}$ & CS & $\begin{array}{c}\text { CUD (DSM-5) } \\
\text { Male: } n=4 \\
\text { Female: } n=2 \\
\text { Mean age: } 36.63 \\
\text { years }\end{array}$ & $n=16$ & rTMS & Left-DLPFC & $\begin{array}{l}20 \text { stimulation } \\
\text { sessions ( } 2 \text { daily, } 5 \\
\text { d/week) for } 2 \\
\text { weeks and } 2 \\
\text { consecutive } \\
\text { maintenance } \\
\text { rTMS sessions } \\
\text { once a week for } 2 \\
\text { weeks. }\end{array}$ & $\begin{array}{l}15 \mathrm{~Hz} \\
100 \%\end{array}$ & NA & $\begin{array}{c}\text { Cocaine use } \\
\text { (urine test) } \\
\text { Cocaine } \\
\text { withdrawal } \\
\text { signs and } \\
\text { symptoms } \\
\text { (CSSA) } \\
\text { Craving (CSSA) } \\
\text { Depression } \\
\text { (BDI) } \\
\text { Anxiety (SAS) } \\
\text { Global Psy- } \\
\text { chopathology } \\
\text { (SCL-90) } \\
\text { Insomnia (ISI) }\end{array}$ & $\begin{array}{c}\text { Baseline, after } 2 \\
\text { and after } 4 \\
\text { weeks of rTMS } \\
\text { treatment. }\end{array}$ \\
\hline $\begin{array}{l}\text { Politi (2008) [46], } \\
\text { Italy }\end{array}$ & CS & $\begin{array}{c}\text { CUD (DSM-IV) } \\
\text { Male: } n=31 \\
\text { Female: } n=5 \\
\text { Mean age: NI }\end{array}$ & $n=36$ & rTMS & Left-DLPFC & $\begin{array}{l}10 \text { daily sessions } \\
\text { of rTMS. }\end{array}$ & $\begin{array}{l}15 \mathrm{~Hz} \\
100 \%\end{array}$ & NA & Craving (VAS) & $\begin{array}{c}\text { During sessions } \\
\text { of rTMS. }\end{array}$ \\
\hline $\begin{array}{c}\text { Rapinesi (2016) } \\
\text { [17] Italy }\end{array}$ & CS & $\begin{array}{c}\text { CUD (DSM-IV) } \\
\text { Male: } n=7 \\
\text { Female: } n=0 \\
\text { Mean age: } 48.71 \\
\text { years }\end{array}$ & $n=7$ & Deep TMS & Bilateral PFC & $\begin{array}{l}3 \text { weekly sessions } \\
\text { on alternate days } \\
\text { for } 4 \text { consecutive } \\
\text { weeks, for a total } \\
\text { of } 12 \text { sessions. }\end{array}$ & $\begin{array}{l}20 \mathrm{~Hz} \\
100 \%\end{array}$ & NA & Craving (VAS) & $\begin{array}{c}\text { Baseline and } \\
\text { after } 2,4 \text { and } 8 \\
\text { weeks of } \\
\text { treatment. }\end{array}$ \\
\hline
\end{tabular}


Table 2. Cont.

\begin{tabular}{|c|c|c|c|c|c|c|c|c|c|c|}
\hline $\begin{array}{c}\text { Author (Year), } \\
\text { Country }\end{array}$ & Study Design & Population & $\begin{array}{c}\text { No. of } \\
\text { Participants }\end{array}$ & Intervention & $\begin{array}{c}\text { Stimulation } \\
\text { Area }\end{array}$ & $\begin{array}{l}\text { Stimulation } \\
\text { Protocol }\end{array}$ & $\begin{array}{c}\text { Frequency (Hz) } \\
\text { and Intensity } \\
(\% \text { RMT) }\end{array}$ & Comparator & Outcomes & Measures \\
\hline $\begin{array}{l}\text { Sanna (2019) } \\
\text { [47], Italy }\end{array}$ & $\mathrm{nRCT}$ & $\begin{array}{c}\text { CUD (DSM-5) } \\
\text { Male: } n=45 \\
\text { Female: } n=2 \\
\text { Mean age: } 37.40 \\
\text { years }\end{array}$ & $n=47$ & iTBS & PFC & $\begin{array}{l}20 \text { stimulations } \\
\text { over } 4 \text { weeks: } 10 \\
\text { stimulations } \\
\text { during the } 1 \text { st } \\
\text { week, } 4 \\
\text { stimulations } \\
\text { during the 2nd } \\
\text { week, } 3 \\
\text { stimulations } \\
\text { during the 3rd and } \\
\text { 4th week. }\end{array}$ & $\begin{array}{c}\text { HF rTMS: } \\
15 \mathrm{~Hz} ; 100 \% \\
\text { iTBS: } \\
5 \mathrm{~Hz} ; 80 \%\end{array}$ & HF rTMS & $\begin{array}{l}\text { Cocaine use } \\
\text { (urine test) } \\
\text { Craving } \\
\text { (CCQ-brief) } \\
\text { Risk for } \\
\text { developing } \\
\text { problems due to } \\
\text { the use of } \\
\text { cocaine } \\
\text { (ASSIST) }\end{array}$ & $\begin{array}{l}\text { Baseline, weekly } \\
\text { during } \\
\text { treatment and at } \\
\text { the end of } \\
\text { treatment. }\end{array}$ \\
\hline $\begin{array}{l}\text { Steele (2019) } \\
\text { [48], USA }\end{array}$ & CS & $\begin{array}{c}\text { CUD (DSM-5) } \\
\text { Male: } n=13 \\
\text { Female: } n=6 \\
\text { Mean age: } 47.4 \\
\text { years }\end{array}$ & $n=19$ & iTBS & Left-DLPFC & $\begin{array}{l}3 \text { iTBS sessions per } \\
\text { day, with an } \\
\text { interval of } \\
\text { approximately } \\
60 \text {-min between } \\
\text { sessions, for } 10 \\
\text { days over a } \\
\text { 2-week period (30 } \\
\text { total iTBS } \\
\text { sessions). }\end{array}$ & $\begin{array}{c}5 \mathrm{~Hz} \\
90-120 \%\end{array}$ & NA & $\begin{array}{l}\text { Amount of } \\
\text { money spent on } \\
\text { cocaine } \\
\text { consumption } \\
\text { Craving (CCS } \\
\text { and CCQ) } \\
\text { iTBS side effects } \\
\text { Depression } \\
\text { (MADRS) }\end{array}$ & $\begin{array}{l}\text { Baseline, during, } \\
\text { and after the } \\
\text { intervention and } \\
\text { at 1-and 4-week } \\
\text { follow-up visits. }\end{array}$ \\
\hline $\begin{array}{l}\text { Terraneo (2016) } \\
\text { [13], Italy }\end{array}$ & $\mathrm{RCT}$ & $\begin{array}{c}\text { CUD } \\
\text { Male: } n=30 \\
\text { Female: } n=2 \\
\text { Mean age: } 40.28 \\
\text { years }\end{array}$ & $n=32$ & rTMS & Left-DLPFC & $\begin{array}{l}1 \text { rTMS session per } \\
\text { day during the } \\
\text { first } 5 \text { days of } \\
\text { treatment, and } \\
\text { then once a week } \\
\text { for the following } 3 \\
\text { weeks, for a total } \\
\text { of } 8 \text { rTMS } \\
\text { sessions. }\end{array}$ & $\begin{array}{l}15 \mathrm{~Hz} \\
100 \%\end{array}$ & $\begin{array}{c}\text { Pharmacological } \\
\text { agents }\end{array}$ & $\begin{array}{c}\text { Cocaine use } \\
\text { (urine test) } \\
\text { Craving (VAS) } \\
\text { Adverse events } \\
\text { Depression } \\
\text { (SCL-90) }\end{array}$ & $\begin{array}{l}\text { Baseline, after } \\
\text { 29-day } \\
\text { treatment and } \\
\text { after 63-day } \\
\text { follow-up. }\end{array}$ \\
\hline
\end{tabular}

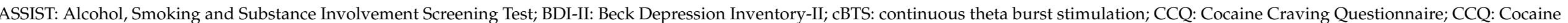

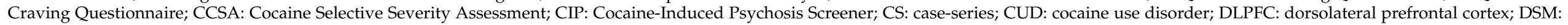

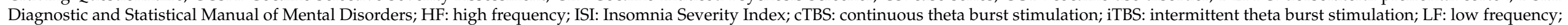

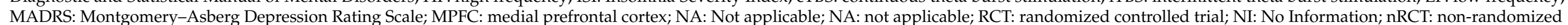

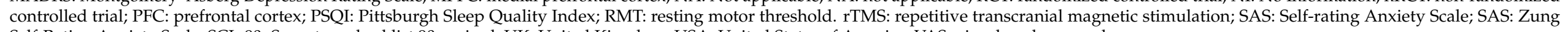
Self-Rating Anxiety Scale; SCL-90: Symptom checklist 90-revised; UK: United Kingdom; USA: United States of America; VAS: visual analogue scale. 


\subsection{Quality Assessment}

\subsubsection{RCTs}

The overall risk of bias was assessed with some concerns, except in one study [44], which enlisted overall high risk of bias. This is mainly because this study did not provide enough details on randomization, blinding process and selection of the reported results. Potential bias due to deviations from the intended intervention and selection of the reported results were the main source of bias. Figure 2 shows the risk of bias summary, with judgements about each risk of bias item for each included study. Figure 3 shows a graph with review authors' judgements about each item presented as percentages across all included studies.

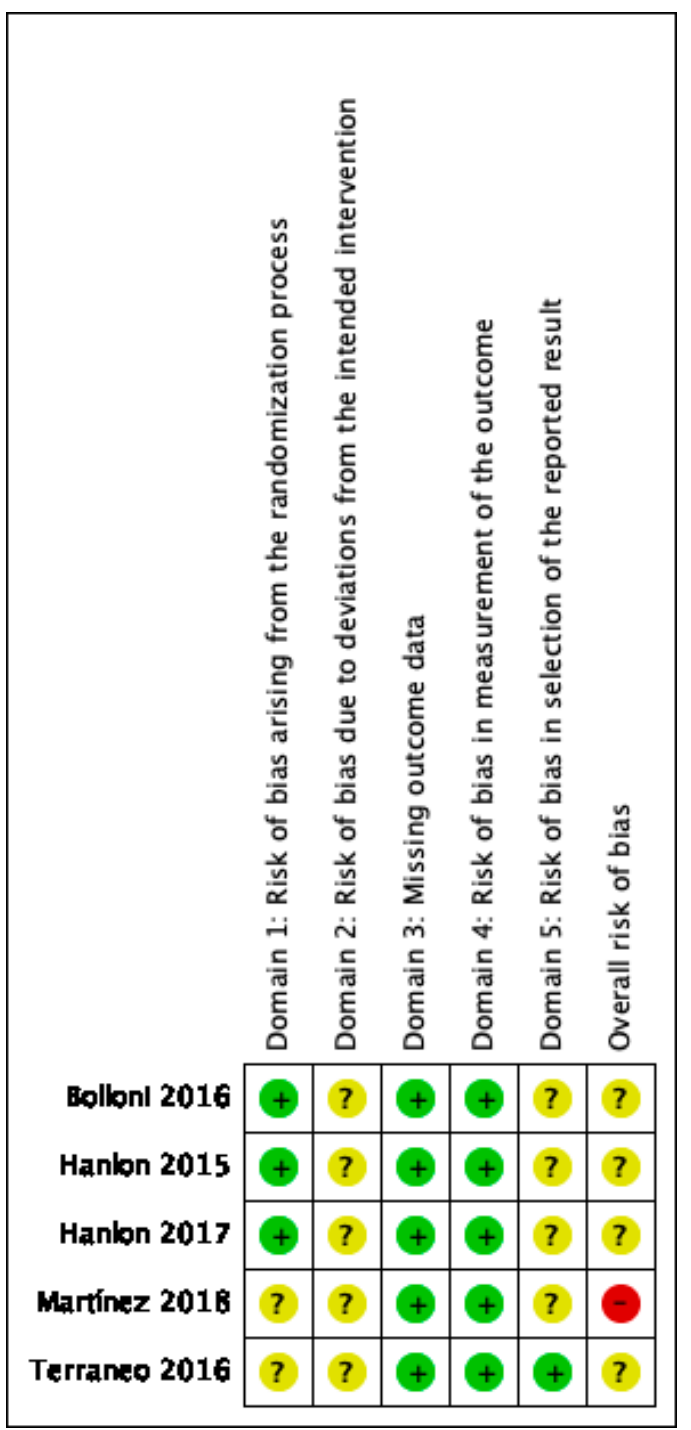

Figure 2. Risk of bias summary. 


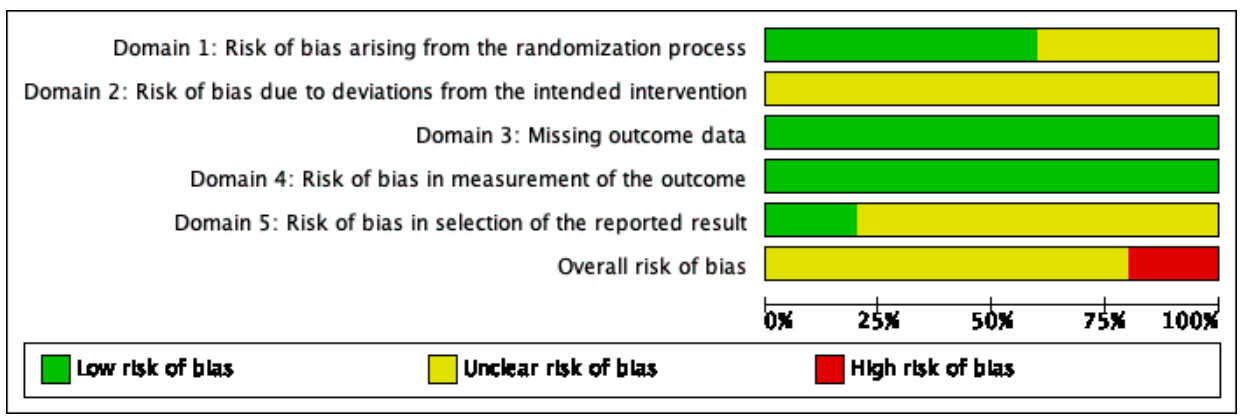

Figure 3. Authors' judgements about each item.

\subsection{2. nRCTs}

The methodological quality of this study [47] was rated as high, since it met eight of nine criteria according to the JBI checklist. Only the one referring to multiple measurements before and after the intervention was negatively rated. The complete quality assessment of the included nRCT can be found in Table S4.

\subsubsection{Case-Series}

The methodological quality was rated as low in one study [46], high in another one [45] and moderate in the remaining four $[17,20,43,48]$. The study rated with lower quality was reported in a letter to the editor and therefore some information was incomplete or uncertain. Overall, the domains with the lowest scores were obtained in aspects related to the collection of cases in more than one centre, the recruitment of consecutive patients, the same level of disease within the participants, and the adequate reporting of losses during follow-up, which the studies did not provide information on. The complete quality assessment of the included case-series can be seen in Table S5.

\subsection{Effectiveness of TMS}

Table 3 shows the results on effectiveness of the included studies.

\subsubsection{Dorsolateral Prefrontal Cortex Stimulation}

rTMS over the left-DLPFC, one RCT [13] and 4 case-series [20,43,45,46] applied high frequency stimulation $(15 \mathrm{~Hz})$, with intensity set at $100 \%$ of the resting motor threshold (RMT) and 2400 pulses per session, although with different numbers of sessions and timing. Another case-series [48] used an iTBS stimulation protocol, with 600 pulses per session and intensity set at $100 \%$.

In Terraneo et al. (2016) [13] $(n=32)$ participants were randomized to rTMS (8 sessions over 4 weeks) or pharmacological treatment. At the end of the treatment period, there were more patients without relapses (no positive urine analysis) in the intervention group: 11 $(69 \%)$ vs. $3(19 \%)(\mathrm{OR}=6.47$, IC95\%: 1.14-36.6). Craving was also significantly lower with rTMS $(p=0.038)$, while no significant differences were found in depression. 
Table 3. Effectiveness results among the included studies.

\begin{tabular}{|c|c|c|c|c|c|c|}
\hline & Cocaine Use & Craving & Anxiety & Depression & Psychopathology & Insomnia \\
\hline \multicolumn{7}{|c|}{ rTMS over the left-DLPFC } \\
\hline $\begin{array}{l}\text { Terraneo et al., } 2016[13] \\
\qquad(n=36) \\
\text { RCT }\end{array}$ & $\begin{array}{c}\text { Negative urine test } \\
\text { during treatment: } \\
\text { rTMS: } 11(69 \%) \\
\text { PT: } 3(19 \%) \\
\text { OR = } 6.47(\text { IC } 95 \%: 1.14, \\
\text { 36.6). }\end{array}$ & $\begin{array}{c}\text { VAS 0-10: } \\
\text { Significantly lower } \\
\text { craving with rTMS } \\
\text { ANOVA RM: } \\
\text { F }(1,27)=4.74, p=0.038\end{array}$ & - & - & - & - \\
\hline $\begin{array}{c}\text { Gómez et al., } 2020[20] \\
(n=87) \\
\text { CS }\end{array}$ & $\begin{array}{c}\text { Days of cocaine use } \\
\text { (mean): reduction at } 30 \\
\text { days: } \\
-18.7(97.3 \%) p<0.001 \\
\text { reduction at } 90 \text { days: } \\
-18.3(95.6 \%) p<0.001\end{array}$ & $\begin{array}{c}\text { CCQ } \\
\text { reduction at } 30 \text { days: } \\
-11.32(89.3 \%) p<0.001 \\
\text { reduction at } 90 \text { days: } \\
-8.86(69.9 \%) p<0.001\end{array}$ & $\begin{array}{c}\text { SAS } \\
\text { reduction at } 30 \text { days: } \\
-11.96(24.9 \%) p<0.001 \\
\text { reduction at } 90 \text { days: } \\
-9.83(20.5 \%) p<0.001\end{array}$ & $\begin{array}{c}\text { BDI-II } \\
\text { reduction at } 30 \text { days: } \\
-13.89(73.1 \%) p<0.001 \\
\text { reduction at } 90 \text { days: } \\
-12.26(64.5 \%) p<0.001\end{array}$ & $\begin{array}{c}\text { SCL-90-R } \\
\text { reduction at } 30 \text { days: } \\
-18.24(27.7 \%) p<0.001 \\
\text { reduction at } 90 \text { days: } \\
-19.45(29.5 \%) p<0.001\end{array}$ & $\begin{array}{c}\text { PSQI } \\
\text { reduction at } 30 \text { days: } \\
-4.24(45.6 \%) p<0.001 \\
\text { reduction at } 90 \text { days: } \\
-3.12(33.8 \%)\end{array}$ \\
\hline $\begin{array}{c}\text { Pettorruso et al., } 2019 \\
\text { [47] }(n=20) \\
\text { CS }\end{array}$ & $\begin{array}{l}\text { Negative urine test at the } \\
\text { end of treatment: } \\
9 \text { of } 16(56.25 \%) \\
(Z=-3.00 ; p=0.003) .\end{array}$ & $\begin{array}{l}\text { CSSA (craving) } \\
\text { reduction at } 4 \text { weeks: } \\
-1.5(33.9 \%) p=0.020\end{array}$ & $\begin{array}{c}\text { SAS } \\
\text { reduction at } 4 \text { weeks: } \\
-8.4(23.0 \%) p=0.001\end{array}$ & $\begin{array}{c}\text { BDI-II } \\
\text { reduction at } 4 \text { weeks: } \\
-9.8(57.1 \%) p=0.008\end{array}$ & $\begin{array}{c}\text { SCL-90-R } \\
\text { reduction at } 4 \text { weeks: } \\
-0.51(52.0 \%) p<0.001\end{array}$ & $\begin{array}{c}\text { ISI } \\
\text { reduction at } 4 \text { weeks: } \\
-5.2(59.7 \%) p=0.077\end{array}$ \\
\hline $\begin{array}{l}\text { Politi et al., } 2008[48] \\
\qquad(n=36) \\
\text { CS }\end{array}$ & - & $\begin{array}{c}\text { Greater reduction with } \\
\text { TMS ANOVA RM (time } \\
\text { effect) } \\
\text { F }(30,270)=4.96 \\
p<0.001\end{array}$ & - & - & - & - \\
\hline
\end{tabular}


Table 3. Cont

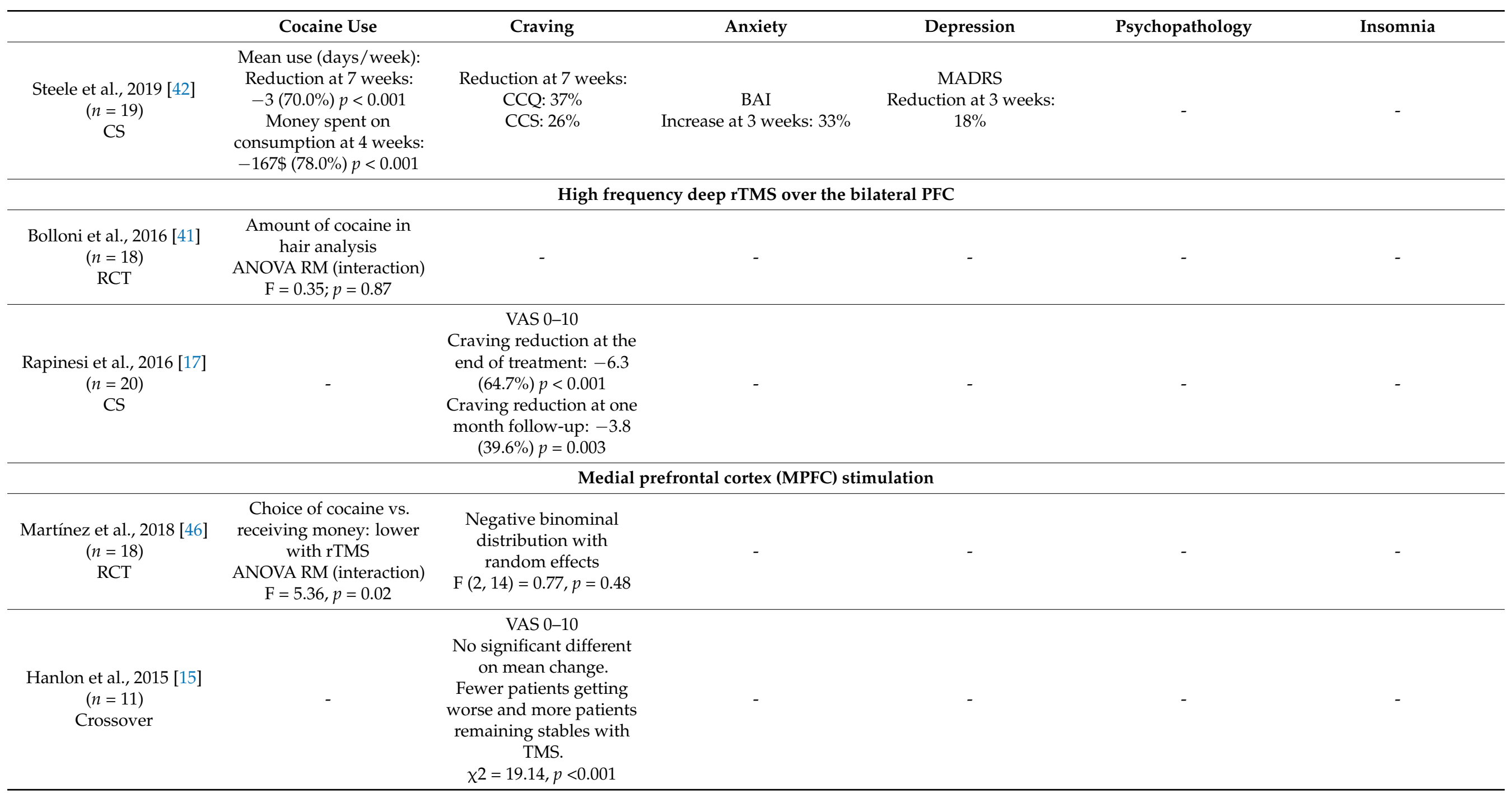


Table 3. Cont

\begin{tabular}{|c|c|c|c|c|c|c|}
\hline & Cocaine Use & Craving & Anxiety & Depression & Psychopathology & Insomnia \\
\hline $\begin{array}{l}\text { Hanlon et al., } 2017 \text { [44] } \\
(n=25) \\
\text { Crossover }\end{array}$ & - & $\begin{array}{c}\text { VAS 0-10 } \\
\text { Post-treatment result not } \\
\text { significant }(p \text {-value not } \\
\text { reported): } \\
\text { rTMS: } 2.93(2.78) \\
\text { Control: } 2.90(2.25)\end{array}$ & - & - & - & - \\
\hline \multicolumn{7}{|c|}{ Comparison between bilateral deep stimulation protocols: iTBS vs. high frequency rTMS } \\
\hline $\begin{array}{l}\text { Sanna et al., } 2019[41] \\
\qquad(n=49) \\
\text { nRCT }\end{array}$ & $\begin{array}{c}\text { Urine test and } \\
\text { consumption statement } \\
\text { ANOVA RM: } \\
\text { Significant effect of time } \\
(\mathrm{F}=49.97 ; p<0.001) \text { but } \\
\text { not of treatment } \\
(\mathrm{F}=0.67) \text { or interaction } \\
(\mathrm{F}=0.66) .\end{array}$ & $\begin{array}{c}\text { brief modified CCQ } \\
\text { ANOVA RM: } \\
\text { Significant effect of time } \\
(\mathrm{F}=127.3 ; p<0.001 \text { but } \\
\text { not of treatment } \\
(\mathrm{F}=1.48) \text { or interaction } \\
(\mathrm{F}=0.03) .\end{array}$ & - & - & - & - \\
\hline
\end{tabular}

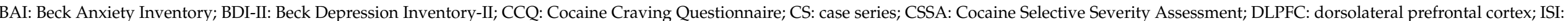

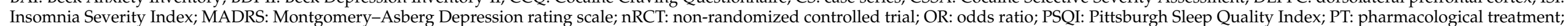

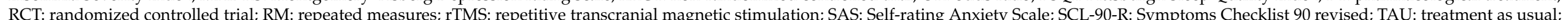
VAS: visual analogue scale. 
Madeo et al. (2020) [43] and Gómez et al. (2020) [20], from the same research group as Terraneo et al. [13], in two different retrospective studies, used an intensified version of the protocol used by the latter (10 sessions in the first five days, and subsequently two weekly sessions for 11 and 12 weeks, respectively). Cocaine consumption was assessed by a combination of urine analysis, self- and hetero report. Gómez et al. (2020) [20] ( $n=87)$ observed a significant reduction in the number of days of consumption at the end of the treatment (from 19.2 to $0.8, \mathrm{t}=12.7, p<0.001$ ). This difference appeared from day 30 . The same pattern of reduction was found in craving (Cocaine Craving Questionnaire, CCQ), sleep quality (Pittsburgh Sleep Quality Inventory, PSQI), depression (Beck Depression Inventory, BDI-II), anxiety (Self-rating Anxiety Scale, SAS) and global psychopathology (Symptoms Checklist-90-Revised, SCL-90-R) (all $p$-values $<0.001)$. Madeo et al. [43] $(n=284)$ retrospectively analysed patients followed for a range of 4 to 989 days. After receiving the intensified protocol, patients were treated with rTMS based on relapses or craving increase. The median time to relapse was 91 days (IC95\%: 70-109), compared to 51 days (IC95\%: $39-78)$ observed in a historical control group treated as usual $(n=173)$ (no statistical contrasts were performed).

Pettorruso et al. (2019) [45] ( $n=20)$ applied 24 sessions, 20 during the first two weeks, and two weekly sessions for the remaining two weeks. Out of the 16 participants who completed the treatment, $9(56.2 \%)$ showed negative urine analysis $(p=0.003)$. Significant reductions were also obtained for craving (subscale of the Cocaine Selective Severity Assessment, CSSA), withdrawal symptoms (total score of the CSSA,), anxiety (SAS), depression (BDI-II), and global psychopathology (SCL-90- R) (all $p$-values $<0.02$ ). Results did not reach the significance level for insomnia severity ( $p=0.077$, Insomnia Severity Index, ISI).

Politi et al. (2008) [46] $(n=36)$ applied 10 sessions, obtaining a significant reduction in craving for consumption $(\mathrm{F}=4.96 ; p<0.001)$.

Finally, Steele et al. (2019) [48] $(n=19)$ used an iTBS stimulation protocol. Treatment took place in three sessions per day, with approximately a 60-min interval between sessions, for 10 days over a two-week period (30 total iTBS sessions). Only 9 participants finished treatment and were followed for four weeks. According to self-reported measures, the weekly amount of money spent on cocaine and the number of days of consumption were reduced by $78 \%(p<0.001)$ and $70 \%(p<0.001)$, respectively. Craving was reduced by $37 \%$ when measured by the Cocaine Craving Questionnaire, and 26\% when measured by the Cocaine Craving Scale. One week after treatment, scores in depression (MontgomeryAsberg Depression Rating Scale) were reduced by 18\%, but a 33\% increase was found in anxiety (Beck Anxiety Inventory), although scores were low in both cases.

High frequency deep rTMS over the bilateral PFC. A sham-controlled trial [41] $(n=18)$ and a case series [17] $(n=7)$ applied deep rTMS using a H1-coil. The stimulation was applied bilaterally, although with a preference for the left hemisphere. The intensity was set at $100 \%$, and frequency was $10 \mathrm{~Hz}$ [41] and $20 \mathrm{~Hz}$ [17], respectively. In both studies, three weekly sessions were applied on alternate days for four weeks.

In Bolloni et al. (2016) [41], the effect on consumption reduction (evaluated by hair analysis) up to 6 months after treatment started was not significant $(\mathrm{F}=0.35 ; p=0.87)$. When groups were analysed separately, only the intervention group showed a significant reduction compared to baseline. Rapinesi et al. (2016) [17] observed a significant reduction in craving at the end of the treatment period (VAS $0-10, p<0.001$ ) and also four weeks later $(p=0.003)$.

\subsubsection{Medial Prefrontal Cortex (MPFC) Stimulation}

Martínez et al. (2018) [44] $(n=18)$ randomized participants into three groups: high frequency deep stimulation (H7-coil), low frequency or sham stimulation. High frequency stimulation was delivered at $10 \mathrm{~Hz}$ and 1200 pulses per session. Low frequency was delivered using a standard $1 \mathrm{~Hz}$ protocol including 900 pulses per session. For the sham condition, a sham coil was present in the same TMS helmet. The intensity was progressively increased in both stimulation groups from $90 \%$ to $110 \%$ of the individual RMT. A choice 
test between administering cocaine or receiving money was performed at baseline, after four sessions and at the end of the treatment period. A significant interaction of treatment by occasion ( $F=5.36, p=0.02)$ was observed. There was little change in cocaine selfadministration in the sham group or in the low frequency group across the three sessions. Only the high frequency group showed a decrease in the choice for cocaine, and this effect was manifested from the third session. However, craving, evaluated with a visual analogue scale, was not affected by any rTMS condition.

Two sham-controlled trials by Hanlon et al. $(2015,2017)[15,42]$ used a cTBS protocol ( $n=11$ and $n=25$, respectively). A single session of cTBS and another session of sham stimulation were cross-applied, separated by 7-14 days. A total of 3600 pulses were applied over the MPFC, with intensity set at $110 \%$ of the individual RMT. Craving was assessed immediately after each session (VAS 0-10), while participants were exposed to cocainerelated cues. No significant differences were observed in both studies. In Hanlon et al. (2015) [15], when the change was analysed categorically (i.e., increase, decrease or no change), significantly fewer participants in the real stimulation session increased craving $\left(\chi^{2}=5.64 ; p=0.05\right)$.

\subsubsection{Comparison between Bilateral Deep Stimulation Protocols: iTBS vs. High Frequency rTMS}

Sanna et al. (2019) [47], in a non-randomized trial $(n=47)$, compared bilateral deep stimulation ( $\mathrm{H} 4$ coil) over the PFC and insula with two different protocols. One group received iTBS (600 pulses/session, $80 \%$ of the individual RMT) while the other group was treated with high-frequency rTMS $(15 \mathrm{~Hz}, 2400$ pulses/session, 100\% of the individual RMT). In both groups, 20 sessions were applied for four weeks, with decreasing frequency. The results showed that the two stimulation protocols significantly reduced consumption measured by urine analysis ( $p<0.001$ for the effect of time). No significant differences were found between protocols. At the end of the treatment period, $82 \%$ and $80 \%$ of patients on iTBS and rTMS, respectively, tested negative in the urine analysis. Craving (Weiss modified Cocaine Craving Questionnaire) and the risk of developing problems due to the use of cocaine (Alcohol, Smoking and Substance Involvement Screening Test, ASSIST) showed the same pattern of results.

\subsection{Evidence on the Safety of TMS for the Treatment of Cocaine Addiction}

Table 4 describes dropouts and adverse events observed among the included studies.

Drop-outs were relatively frequent in around 20\% of the studies, although none specified that they were due to safety problems. Adverse events were not serious in any case.

Two studies provided detailed data on adverse events. In Madeo et al. (2020) [43], one case of seizure occurred in a 27-year-old woman 66 days after the first rTMS session. Another case of a hypomanic episode was reported in a 37-year-old man, just under 90 days after his first rTMS session. Twenty-three patients (8\%) reported headache after stimulation, while the rest of the events, mild and transient, occurred in one or two patients each. In Steele et al. (2019) [48], one participant suffered right-hand supination/pronation at the wrist 10-15 min after the iTBS session. Two weeks after the iTBS termination, this same participant reported visual illusions and tactile hallucinations, which developed slowly over several days but were cleared promptly with a single dose of olanzapine. Nine of the 14 participants $(64,3 \%)$ experienced at least one headache, usually beginning during or shortly after iTBS. 
Table 4. Dropouts and adverse events.

\begin{tabular}{|c|c|c|}
\hline Author (Year) & Drop-Outs & Adverse Events \\
\hline Boloni (2016) [41] & $4 / 18(22.2 \%)$ & $\begin{array}{l}\text { Discomfort was not observed except for a patient who suffered from a mild } \\
\text { headache after receiving active stimulation. }\end{array}$ \\
\hline Gómez (2020) [20] & NR & $\begin{array}{c}\text { Serious AEs were not reported. There were no seizures, syncopes, neurological } \\
\text { complications or subjective complaints about memory or concentration } \\
\text { impairment limiting the treatment. }\end{array}$ \\
\hline Hanlon (2015) [15] & NA & NR \\
\hline Hanlon (2017) [42] & NA & NR \\
\hline Madeo (2020) [43] & $58 / 284(20.4 \%)$ & $\begin{array}{l}\text { AEs were reported by } 41 \text { of the } 284 \text { patients. AEs reported were headache } \\
(n=23) \text {, hypomania }(n=4) \text {, anxiety }(n=2) \text {, irritability }(n=2) \text {, dental pain } \\
(n=2), \text { scalp discomfort during the first } 2(n=1) \text {, angioedema and urticaria } \\
(n=1) \text {, distractibility }(n=1) \text {, dizziness }(n=1) \text {, nausea }(n=1) \text {, nausea and } \\
\text { numbness }(n=1) \text {, seizure }(n=1) \text {, and a hypomanic episode }(n=1) .\end{array}$ \\
\hline Martínez (2018) [44] & NR & $\begin{array}{l}\text { Participants had difficulty tolerating stimulation that increased from } 100 \text { to } \\
120 \% \text { of MT, and thus the protocol was amended by lowering the maximal } \\
\text { stimulation. }\end{array}$ \\
\hline Pettorruso (2019) [45] & $4 / 20(20 \%)$ & The treated subjects reported no significant side effect. \\
\hline Politi (2008) [46] & NR & NR \\
\hline Rapinesi (2016) [17] & $0 / 7(0 \%)$ & All patients tolerated the stimulation without complications or AEs. \\
\hline Sanna (2019) [47] & $4 / 47(8.5 \%)$ & $\begin{array}{l}\text { A few participants in both the } 15 \mathrm{~Hz} \text { rTMS and the iTBS groups reported mild } \\
\text { discomfort at the start of stimulation, especially during the first session. Both } \\
\text { treatments were safe and there were no serious or unexpected AEs related to } \\
\text { the treatments. There were no seizures or any other transient neurological } \\
\text { event. }\end{array}$ \\
\hline Steele (2019) [48] & $7 / 16(43.7 \%)$ & $\begin{array}{l}\text { There were no unexpected, serious AEs. Nine of the } 14 \text { participants } \\
\text { experienced at least one headache. One participant experienced sudden pain } \\
\text { around her eyes and one experienced muscle soreness in the right forearm. No } \\
\text { negative side-effects in cognitive and affective assessments were reported. No } \\
\text { participant experienced any signs of mania or suicidality. After completing } 26 \\
\text { iTBS sessions, a participant reported right-hand supination/pronation and } \\
\text { thus treatment was terminated. }\end{array}$ \\
\hline Terraneo (2016) [13] & $3 / 32(9.4 \%)$ & $\begin{array}{l}\text { A few participants reported mild discomfort at the start of stimulation, } \\
\text { especially during the first session, but overall, there were no significant } \\
\text { differences in AEs across groups. There were no serious or unexpected AEs. }\end{array}$ \\
\hline \multicolumn{3}{|c|}{ AEs: adverse events; NR: not reported; NA: not applicable. } \\
\hline
\end{tabular}

\section{Discussion}

CUD is a disease that can cause cognitive dysfunctions at various levels, such as lack of impulse control, drug-seeking compulsions, and inability to modulate behaviours according to the different circumstances [45]. Today it is known that addictions to substances such as cocaine can compromise the activity patterns of the entire brain, and that their effects are focused on meso-cortical alterations and in the activity of dopamine, which affect the centres of motivation and desire to consume the substance [18].

In recent years, non-invasive brain stimulation techniques have provided insights into the neural networks affected by CUD and have been tested as an alternative to addiction treatments [17]. Such is the case of rTMS for which there is currently evidence that indicates a potential benefit in reducing the consumption of alcohol and other drugs [22]. This review makes a pioneering effort to collect evidence on the safety, effectiveness, and cost effectiveness of using different rTMS protocols on the reduction of cocaine consumption and reduction of craving.

What could be the mechanism by which rTMS produces beneficial effects on cocaine consumption? The honest answer is that we do not know, and, in any case, it seems to be 
out of the scope of a review article such as this. However, taking into account data obtained by mean of optogenetic stimulation in a rat model of cocaine addiction [12], it is tempting to speculate on the possibility that the activation of a hypofunctional prefrontal cortex (whose deep-layer pyramidal neurons project to subcortical structures implicated in drugseeking behaviours, including the nucleus accumbens and dorsal striatum), produces a regulation on dysfunctional reward circuits. The results by Chen et al. clearly demonstrate two crucial aspects related to addiction. First, that consumption produces a reduction of activity in the cortex in cocaine-seeking rats and, second, that by increasing the excitability of the cortex, compulsive behaviour decreases. How rat prelimbic cortex stimulation reduces cocaine seeking remains to be resolved. An interesting possibility would be that the activation of the descending glutamatergic connections from the cortex might regulate the dopaminergic activity of accumbens and dorsal striatum. Such dopaminergic activity derives from the inputs arising in the ventral tegmental area and substantia nigra, and in cocaine addiction would have an abnormal dynamic to be corrected. In fact, it has been shown in humans that high-frequency rTMS of the left DLPFC induces dopamine release in the striatum [49]. Furthermore, high-frequency stimulation (rTMS at 20 or $25 \mathrm{~Hz}$ ) delivered on the frontal cortex of rats induces dopamine release throughout the mesolimbic and mesostriatal circuits [50-52]. This suggests that the therapeutic benefit observed in humans using high-frequency TMS over the DLPFC could be related to a regulation of dopamine activity.

Moreover, it has been shown that $12-\mathrm{Hz}$ optogenetic stimulation of medial prefrontal cortex projections to the nucleus accumbens dropped sensitivity to a cocaine challenge in mice. This specific protocol activates metabotropic glutamate receptors, which depotentiates excitatory inputs on dopamine D1 receptors [53].

Regarding safety, no serious adverse effects have been observed [43], and the most common adverse effect was mild and transient headache. These results are supported by the previous literature on rTMS in other clinical conditions (e.g., depression, OCD, other addictions) [22,54].

Although the included studies have reported favourable results in measures of consumption reduction and craving, the heterogeneity in central aspects, such as the evaluation criteria or the follow-ups, make it difficult to summarize and compare the results they offer. Additionally, only five of the 12 studies were RCTs, the rest were a controlled trial and six uncontrolled studies (with a significant percentage of losses in several of them) except in the case of two retrospective studies.

Despite this context of low-quality evidence, available results suggest that highfrequency rTMS applied to the left DLPFC may produce clinically relevant benefits in reducing cocaine use and craving, and possibly in other variables such as depression and insomnia $[13,20,45,47,55]$. As it was mentioned previously, this can be explained because the DLPFC participates in the reward, motivation and decision-making systems that allow inhibitory control $[13,19]$. However, the malfunction of the DLPFC (which may be abnormal in substance use disorders) may explain a reduction in inhibitory control and a greater probability of relapse into alcohol and drug use. Hence, high-frequency rTMS protocols with excitatory effect, located in the DLPFC, can provide better inhibitory control responses, as has been observed in the results of the studies included in this review.

These results are consistent with those reported by other studies in which a single session of repetitive TMS (rTMS) significantly reduced craving for cocaine, a reduction that persisted four hours after the end of the session [56]. Similarly, previous studies with stimulation protocols on bilateral DLPFC in other types of addictions have reported a reduction in nicotine consumption [55] and the desire for alcohol after cycles of deep transcranial magnetic stimulation (dTMS [17,57,58]). The evidence on the effects of other protocols (deep stimulation, TBS) is too limited to draw any conclusions, as is the evidence for stimulation of the medial PFC or other brain locations.

Although the use of TMS involves some difficulties, such as the need to transfer patients to centers that have such equipment, as well as the need to have physicians and 
experts in TMS, and protocols and technicians trained in handling the equipment, it is an option to consider in a scenario where there is a lack of approved treatment, and the fact that $70 \%$ of cocaine users seeking treatment relapse within the first three months, that cocaine dependent people have limited support to overcome this chronic illness [59].

\section{Limitations}

Since not all studies are RCTs, and the fact that the included studies had small sample sizes and a short follow-up, the evidence obtained does not allow conclusive statements.

\section{Conclusions}

In this review, despite the low quality of the studies, significant first results of the efficacy of transcranial magnetic stimulation (TMS) have been observed in terms of the number of doses consumed and the reduction in craving to consume, with respect to the baseline values reported by the participants and by different biological tests (such as urine or hair). The ability to modulate cravings for use in a specific way through non-invasive brain stimulation techniques, such as rTMS, could be a new tool to use as an adjunct to the behavioural treatment of addiction, especially for cocaine use, in that there is currently no specific pharmacotherapy approved for its treatment. That said, in order to consider TMS as a procedure likely to be recommended for the treatment of cocaine addiction, controlled clinical trials are needed carried out under rigorous standards with respect, for example, to the characterization of the participants, the randomization and the blinding procedures. In this regard, there are a number of variables specific to the technique that make it difficult to obtain the "perfect" protocol for each subject and to obtain the best possible results. In the future, it will be necessary to conduct comparative studies to evaluate these key variables, among which it is necessary to mention the target region to be stimulated, the methods to locate the target and the type of coil to be used, the number and frequency of the pulses and the number of sessions.

Supplementary Materials: The following are available online at https:/ /www.mdpi.com/article/10 .3390/jcm10235595/s1, Table S1: PRISMA check-list, Table S2: Search strategy, Table S3: Ongoing Studies about TMS for CUD, Table S4: Quality assessment in nRCT, Table S5: Quality assessment in case-series.

Author Contributions: Conceptualization, Supervision, Writing—Original draft preparation, Writing Reviewing and Editing A.T.-C. (Alezandra Torres-Castaño); Formal analysis, Data curation A.R.-S.; Conceptualization, Writing-Reviewing and Editing, L.P.-P.; Writing-Original draft preparation, Visualization, Writing—Reviewing and Editing, A.D.-D.; Writing—Reviewing and Editing, A.T.-C. (Ana Toledo-Chavarri); Writing-Reviewing and Editing, V.R.-G.; Writing-Reviewing and Editing, Y.Á.-P.; Conceptualization, Writing-Reviewing and Editing, J.C.-M.; Conceptualization, WritingReviewing and Editing, I.P.-G., Conceptualization, Writing-Reviewing and Editing, P.S.-P. All authors have approved the final article. All authors have read and agreed to the published version of the manuscript.

Funding: This research received no external funding.

Institutional Review Board Statement: Not applicable.

Informed Consent Statement: Not applicable.

Data Availability Statement: The data presented in this study are available in supplementary material.

Acknowledgments: We would like to thank Leticia Rodriguez-Rodriguez, Carlos Gonzalez-Rodriguez and Pedro Serrano-Aguilar for their collaboration and support in the preparation of this paper.

Conflicts of Interest: The authors declare no conflict of interest. 


\section{References}

1. European Monitoring Centre for Drugs and Drug addiction Annual Overview of the European Drug Situation on 27 May in a Multilingual, Multimedia Information Package Focusing on Today's Rapidly Shifting Drug Phenomenon, EMCDDA: 2014. Available online: https://www.emcdda.europa.eu/publications/edr/trends-developments/2014_en (accessed on 20 November 2020).

2. United Nations, G.A. World Drug Report 2020. Report 2. Drug Use and Health Consequences. 2020. Available online: https:/ /wdr.unodc.org/wdr2020/field/WDR20_Booklet_2.pdf (accessed on 23 November 2020).

3. Volkow, N.D.; Fowler, J.S.; Wang, G.J.; Swanson, J.M. Dopamine in drug abuse and addiction: Results from imaging studies and treatment implications. Mol. Psychiatry 2004, 9, 557-569. [CrossRef] [PubMed]

4. Moreno-López, L.; Catena, A.; Fernández-Serrano, M.J.; Delgado-Rico, E.; Stamatakis, E.A.; Pérez-García, M.; Verdejo-García, A. Trait impulsivity and prefrontal gray matter reductions in cocaine dependent individuals. Drug Alcohol Depend. 2012, 125, 208-214. [CrossRef] [PubMed]

5. Matochik, J.A.; London, E.D.; Eldreth, D.A.; Cadet, J.L.; Bolla, K.I. Frontal cortical tissue composition in abstinent cocaine abusers: A magnetic resonance imaging study. Neuroimage 2003, 19, 1095-1102. [CrossRef]

6. Goldstein, R.Z.; Volkow, N.D. Dysfunction of the prefrontal cortex in addiction: Neuroimaging findings and clinical implications. Nat. Rev. Neurosci. 2011, 12, 652-669. [CrossRef] [PubMed]

7. Kaufman, J.N.; Ross, T.J.; Stein, E.A.; Garavan, H. Cingulate hypoactivity in cocaine users during a GO-NOGO task as revealed by event-related functional magnetic resonance imaging. J. Neurosci. 2003, 23, 7839-7843. [CrossRef] [PubMed]

8. Volkow, N.D.; Fowler, J.S.; Wang, G.J. The addicted human brain: Insights from imaging studies. J. Clin. Invest. 2003, 111, 1444-1451. [CrossRef]

9. Licata, S.C.; Renshaw, P.F. Neurochemistry of drug action: Insights from proton magnetic resonance spectroscopic imaging and their relevance to addiction. Ann. N. Y. Acad. Sci. 2010, 1187, 148-171. [CrossRef]

10. Ke, Y.; Streeter, C.C.; Nassar, L.E.; Sarid-Segal, O.; Hennen, J.; Yurgelun-Todd, D.A.; Awad, L.A.; Rendall, M.J.; Gruber, S.A.; Nason, A.; et al. Frontal lobe GABA levels in cocaine dependence: A two-dimensional, J-resolved magnetic resonance spectroscopy study. Psychiatry Res.-Neuroimaging 2004, 130, 283-293. [CrossRef]

11. Jasinska, A.J.; Chen, B.T.; Bonci, A.; Stein, E.A. Dorsal medial prefrontal cortex (MPFC) circuitry in rodent models of cocaine use: Implications for drug addiction therapies. Addict. Biol. 2015, 20, 215-226. [CrossRef]

12. Chen, B.T.; Yau, H.J.; Hatch, C.; Kusumoto-Yoshida, I.; Cho, S.L.; Hopf, F.W.; Bonci, A. Rescuing cocaine-induced prefrontal cortex hypoactivity prevents compulsive cocaine seeking. Nature 2013, 496, 359-362. [CrossRef]

13. Terraneo, A.; Leggio, L.; Saladini, M.; Ermani, M.; Bonci, A.; Gallimberti, L. Transcranial magnetic stimulation of dorsolateral prefrontal cortex reduces cocaine use: A pilot study. Eur. Neuropsychopharmacol. 2016, 26, 37-44. [CrossRef]

14. Nutt, D.J.; Lingford-Hughes, A.; Erritzoe, D.; Stokes, P.R.A. The dopamine theory of addiction: 40 years of highs and lows. Nat. Rev. Neurosci. 2015, 16, 305-312. [CrossRef]

15. Hanlon, C.A.; Dowdle, L.T.; Austelle, C.W.; Devries, W.; Mithoefer, O.; Badran, B.W.; George, M.S. What goes up, can come down: Novel brain stimulation paradigms may attenuate craving and craving-related neural circuitry in substance dependent individuals. Brain Res. 2015, 1628, 199-209. [CrossRef] [PubMed]

16. Wilson, S.J.; Sayette, M.A.; Fiez, J.A. Prefrontal responses to drug cues: A neurocognitive analysis. Nat. Neurosci. 2004, 7, 211-214. [CrossRef] [PubMed]

17. Rapinesi, C.; Del Casale, A.; Di Pietro, S.; Ferri, V.R.; Piacentino, D.; Sani, G.; Raccah, R.N.; Zangen, A.; Ferracuti, S.; Vento, A.E.; et al. Add-on high frequency deep transcranial magnetic stimulation (dTMS) to bilateral prefrontal cortex reduces cocaine craving in patients with cocaine use disorder. Neurosci. Lett. 2016, 629, 43-47. [CrossRef] [PubMed]

18. Bolloni, C.; Badas, P.; Corona, G.; Diana, M. Transcranial magnetic stimulation for the treatment of cocaine addiction: Evidence to date. Subst. Abuse Rehabil. 2018, Volume 9, 11-21. [CrossRef]

19. Ferenczi, E.; Deisseroth, K. Illuminating next-generation brain therapies. Nat. Neurosci. 2016, 19, 414-416. [CrossRef]

20. Gómez Pérez, L.J.; Cardullo, S.; Cellini, N.; Sarlo, M.; Monteanni, T.; Bonci, A.; Terraneo, A.; Gallimberti, L.; Madeo, G. Sleep quality improves during treatment with repetitive transcranial magnetic stimulation (rTMS) in patients with cocaine use disorder: A retrospective observational study. BMC Psychiatry 2020, 20, 1-12. [CrossRef]

21. Dakwar, E.; Nunes, E.V.; Hart, C.L.; Foltin, R.W.; Mathew, S.J.; Carpenter, K.M.; Choi, C.J.; Basaraba, C.N.; Pavlicova, M.; Levin, F.R. A single ketamine infusion combined with mindfulness-based behavioral modification to treat cocaine dependence: A randomized clinical trial. Am. J. Psychiatry 2019, 176, 923-930. [CrossRef]

22. Coles, A.S.; Kozak, K.; George, T.P. A review of brain stimulation methods to treat substance use disorders. Am. J. Addict. 2018, 27, 71-91. [CrossRef]

23. Lefaucheur, J.P.; André-Obadia, N.; Antal, A.; Ayache, S.S.; Baeken, C.; Benninger, D.H.; Cantello, R.M.; Cincotta, M.; de Carvalho, M.; De Ridder, D.; et al. Evidence-based guidelines on the therapeutic use of repetitive transcranial magnetic stimulation (rTMS). Clin. Neurophysiol. 2014, 125, 2150-2206. [CrossRef]

24. Barker, A.T.; Jalinous, R.; Freeston, I.L. Non-invasive magnetic stimulation of human motor cortex. Lancet 1985, $11,1106-1107$. [CrossRef]

25. Terao, Y.; Ugawa, Y. Basic mechanisms of TMS. J. Clin. Neurophysiol. 2002, 19, 322-343. [CrossRef] [PubMed] 
26. Deng, Z.D.; Lisanby, S.H.; Peterchev, A.V. Coil design considerations for deep transcranial magnetic stimulation. Clin. Neurophysiol. 2014, 125, 1202-1212. [CrossRef]

27. Roth, Y.; Amir, A.; Levkovitz, Y.; Zangen, A. Three-dimensional distribution of the electric field induced in the brain by transcranial magnetic stimulation using figure-8 and deep H-coils. J. Clin. Neurophysiol. 2007, 24, 31-38. [CrossRef]

28. Cohen, L.G.; Roth, B.J.; Nilsson, J.; Dang, N.; Panizza, M.; Bandinelli, S.; Friauf, W.; Hallett, M. Effects of coil design on delivery of focal magnetic stimulation. Technical considerations. Electroencephalogr. Clin. Neurophysiol. 1990, 75, 350-357. [CrossRef]

29. Chen, R.; Classen, J.; Gerloff, C.; Celnik, P.; Wassermann, E.M.; Hallett, M.; Cohen, L.G. Depression of motor cortex excitability by low-frequency transcranial magnetic stimulation. Neurology 1997, 48, 1398-1403. [CrossRef]

30. Pascual-leone, A.; Valls-solé, J.; Wassermann, E.M.; Hallett, M. Responses to rapid-rate transcranial magnetic stimulation of the human motor cortex. Brain 1994, 117, 847-858. [CrossRef]

31. Daskalakis, Z.J.; Möller, B.; Christensen, B.K.; Fitzgerald, P.B.; Gunraj, C.; Chen, R. The effects of repetitive transcranial magnetic stimulation on cortical inhibition in healthy human subjects. Exp. Brain Res. 2006, 174, 403-412. [CrossRef] [PubMed]

32. Fitzgerald, P.B.; Brown, T.L.; Daskalakis, Z.J. The application of transcranial magnetic stimulation in psychiatry and neurosciences research. Acta Psychiatr. Scand. 2002, 105, 324-340. [CrossRef]

33. Huang, Y.Z.; Edwards, M.J.; Rounis, E.; Bhatia, K.P.; Rothwell, J.C. Theta burst stimulation of the human motor cortex. Neuron 2005, 45, 201-206. [CrossRef]

34. Bulteau, S.; Sébille, V.; Fayet, G.; Thomas-Ollivier, V.; Deschamps, T.; Bonnin-Rivalland, A.; Laforgue, E.; Pichot, A.; Valrivière, P.; Auffray-Calvier, E.; et al. Efficacy of intermittent Theta Burst Stimulation (iTBS) and 10-Hz high-frequency repetitive transcranial magnetic stimulation (rTMS) in treatment-resistant unipolar depression: Study protocol for a randomised controlled trial. Trials 2017, 18. [CrossRef] [PubMed]

35. Rossi, S.; Hallett, M.; Rossini, P.M.; Pascual-Leone, A.; Avanzini, G.; Bestmann, S.; Berardelli, A.; Brewer, C.; Canli, T.; Cantello, R.; et al. Safety, ethical considerations, and application guidelines for the use of transcranial magnetic stimulation in clinical practice and research. Clin. Neurophysiol. 2009, 120, 2008-2039. [CrossRef]

36. Rossi, S.; Antal, A.; Bestmann, S.; Bikson, M.; Brewer, C.; Brockmöller, J.; Carpenter, L.L.; Cincotta, M.; Chen, R.; Daskalakis, J.D.; et al. Safety and recommendations for TMS use in healthy subjects and patient populations, with updates on training, ethical and regulatory issues: Expert Guidelines. Clin. Neurophysiol. 2020, 132. [CrossRef]

37. Page, M.J.; McKenzie, J.E.; Bossuyt, P.M.; Boutron, I.; Hoffmann, T.C.; Mulrow, C.D.; Shamseer, L.; Tetzlaff, J.M.; Akl, E.A.; Brennan, S.E.; et al. The PRISMA 2020 statement: An updated guideline for reporting systematic reviews. BMJ $2021,372$.

38. Sterne, J.A.C.; Savović, J.; Page, M.J.; Elbers, R.G.; Blencowe, N.S.; Boutron, I.; Cates, C.J.; Cheng, H.Y.; Corbett, M.S.; Eldridge, S.M.; et al. RoB 2: A revised tool for assessing risk of bias in randomised trials. BMJ 2019, 366. [CrossRef]

39. Tufanaru, C.; Munn, Z.; Aromataris, E.; Campbell, J.H.L. Chapter 3: Systematic reviews of effectiveness. In Manual for Evidence Synthesis; Aromataris, E., Munn, Z., Eds.; JBI: Adelaide, Australia, 2020.

40. Institute of Health Economics (IHE). Quality Appraisal of Case Series Studies Checklist. 2014. Available online: https://cdnlinks.lww.com/permalink/jbjsrev/a/jbjsrev_2018_03_28_greysdc_17-00129_sdc2.pdf (accessed on 20 November 2020).

41. Bolloni, C.; Panella, R.; Pedetti, M.; Frascella, A.G.; Gambelunghe, C.; Piccoli, T.; Maniaci, G.; Brancato, A.; Cannizzaro, C.; Diana, M. Bilateral Transcranial Magnetic Stimulation of the Prefrontal Cortex Reduces Cocaine Intake: A Pilot Study. Front. Psychiatry 2016, 7, 1. [CrossRef]

42. Hanlon, C.A.; Dowdle, L.T.; Correia, B.; Mithoefer, O.; Kearney-Ramos, T.; Lench, D.; Griffin, M.; Anton, R.F.; George, M.S. Left frontal pole theta burst stimulation decreases orbitofrontal and insula activity in cocaine users and alcohol users. Drug Alcohol Depend. 2017, 178, 310-317. [CrossRef] [PubMed]

43. Madeo, G.; Terraneo, A.; Cardullo, S.; Gómez Pérez, L.J.; Cellini, N.; Sarlo, M.; Bonci, A.; Gallimberti, L. Long-Term Outcome of Repetitive Transcranial Magnetic Stimulation in a Large Cohort of Patients With Cocaine-Use Disorder: An Observational Study. Front. Psychiatry 2020, 11, 1-8. [CrossRef]

44. Martinez, D.; Urban, N.; Grassetti, A.; Chang, D.; Hu, M.C.; Zangen, A.; Levin, F.R.; Foltin, R.; Nunes, E.V. Transcranial magnetic stimulation of medial prefrontal and cingulate cortices reduces cocaine self-administration: A pilot study. Front. Psychiatry 2018, 9, 10-15. [CrossRef]

45. Pettorruso, M.; Martinotti, G.; Santacroce, R.; Montemitro, C.; Fanella, F.; di Giannantonio, M. rTMS Reduces Psychopathological Burden and Cocaine Consumption in Treatment-Seeking Subjects With Cocaine Use Disorder: An Open Label, Feasibility Study. Front. Psychiatry 2019, 10, 1-9. [CrossRef]

46. Politi, E.; Fauci, E.; Santoro, A.; Smeraldi, E. Daily sessions of transcranial magnetic stimulation to the left prefrontal cortex gradually reduce cocaine craving. Am. J. Addict. 2008, 17, 345-346. [CrossRef] [PubMed]

47. Sanna, A.; Fattore, L.; Badas, P.; Corona, G.; Cocco, V.; Diana, M. Intermittent Theta Burst Stimulation of the Prefrontal Cortex in Cocaine Use Disorder: A Pilot Study. Front. Neurosci. 2019, 13, 765. [CrossRef] [PubMed]

48. Steele, V.R.; Maxwell, A.M.; Ross, T.J.; Stein, E.A.; Salmeron, B.J. Accelerated intermittent theta-burst stimulation as a treatment for cocaine use disorder: A proof-of-concept study. Front. Neurosci. 2019, 13, 1-13. [CrossRef]

49. Strafella, A.P.; Paus, T.; Barrett, J.; Dagher, A. Repetitive transcranial magnetic stimulation of the human prefrontal cortex induces dopamine release in the caudate nucleus. J. Neurosci. 2001, 21. [CrossRef] 
50. Keck, M.E.; Welt, T.; Müller, M.B.; Erhardt, A.; Ohl, F.; Toschi, N.; Holsboer, F.; Sillaber, I. Repetitive transcranial magnetic stimulation increases the release of dopamine in the mesolimbic and mesostriatal system. Neuropharmacology 2002, 43, 101-109. [CrossRef]

51. Kanno, M.; Matsumoto, M.; Togashi, H.; Yoshioka, M.; Mano, Y. Effects of acute repetitive transcranial magnetic stimulation on dopamine release in the rat dorsolateral striatum. J. Neurol. Sci. 2004, 217, 73-81. [CrossRef] [PubMed]

52. Zangen, A.; Hyodo, K. Transcranial magnetic stimulation induces increases in extracellular levels of dopamine and glutamate in the nucleus accumbens. Neuroreport 2002, 13, 2401-2405. [CrossRef]

53. Creed, M.; Pascoli, V.J.; Lüscher, C. Addiction therapy. Refining deep brain stimulation to emulate optogenetic treatment of synaptic pathology. Science 2015, 347, 659-664. [CrossRef]

54. Enokibara, M.; Trevizol, A.; Shiozawa, P.; Cordeiro, Q. Establishing an Effective TMS Protocol for Craving in Substance Addiction: Is It Possible? Am. J. Addict. 2016, 25, 28-30. [CrossRef]

55. Dinur-Klein, L.; Dannon, P.; Hadar, A.; Rosenberg, O.; Roth, Y.; Kotler, M.; Zangen, A. Smoking cessation induced by deep repetitive transcranial magnetic stimulation of the prefrontal and insular cortices: A prospective, randomized controlled trial. Biol. Psychiatry 2014, 76, 742-749. [CrossRef]

56. Camprodon, J.A.; Martínez-Raga, J.; Alonso-Alonso, M.; Shih, M.C.; Pascual-Leone, A. One session of high frequency repetitive transcranial magnetic stimulation (rTMS) to the right prefrontal cortex transiently reduces cocaine craving. Drug Alcohol Depend. 2007, 86, 91-94. [CrossRef]

57. Rapinesi, C.; Kotzalidis, G.D.; Serata, D.; Del Casale, A.; Bersani, F.S.; Solfanelli, A.; Scatena, P.; Raccah, R.N.; Brugnoli, R.; Digiacomantonio, V.; et al. Efficacy of add-on deep transcranial magnetic stimulation in comorbid alcohol dependence and dysthymic disorder: Three case reports. Prim. Care Companion J. Clin. Psychiatry 2013, 15. [CrossRef] [PubMed]

58. Girardi, P.; Rapinesi, C.; Chiarotti, F.; Kotzalidis, G.D.; Piacentino, D.; Serata, D.; Del Casale, A.; Scatena, P.; Mascioli, F.; Raccah, R.N.; et al. Add-on deep transcranial magnetic stimulation (dTMS) in patients with dysthymic disorder comorbid with alcohol use disorder: A comparison with standard treatment. World J. Biol. Psychiatry 2015, 16, 66-73. [CrossRef] [PubMed]

59. Kearney-Ramos, T.E.; Dowdle, L.T.; Mithoefer, O.J.; Devries, W.; George, M.S.; Hanlon, C.A. State-dependent effects of ventromedial prefrontal cortex continuous thetaburst stimulation on cocaine cue reactivity in chronic cocaine users. Front. Psychiatry 2019, 10, 1-10. [CrossRef] [PubMed] 